\title{
QUASI $(s, r)$-CONTRACTIVE MULTI-VALUED OPERATORS ON b-METRIC SPACE AND RELATED FIXED POINT THEOREMS
}

\author{
EI EI NYEIN ${ }^{1}$, AUNG KHAING ZAW ${ }^{2}$ \\ ${ }^{1,2}$ School of Mathematics and Statistics, Beijing Institute of Technology, \\ Beijing, 100081, China, \\ E-mail: eieinyein1985@yahoo.com, akzbee451986@yahoo.com
}

\begin{abstract}
This paper aims to introduce a new type of quasi $(s, r)$-contractive multivalued operator on b-complete metric space and to extend the results for the fixed point theorems of quasi $(s, r)$-contractive multi-valued operator. As an application, the existence of the solution for a type of differential equation is given.

key words: b-metric space; quasi $(s, r)$-contractive multi-valued operator; fixed point theorem.
\end{abstract}

\section{INTRODUCTION}

The theory of multi-valued mappings plays an important role in several branches of pure and applied mathematics because of its many applications, especially, not only in real and complex analysis but also in optimal control problems. Czerwik $[2,3]$ introduced the notion of b-metric spaces and proved the fixed point theorems for single-valued and multi-valued mappings in b-metric spaces. Ciric [9] introduced the single-valued quasi contractive operator in metric space and investigated the existence and uniqueness of the fixed point theorem for quasi contractive operators in 1974. He also introduced quasi contractive multi-valued operator and established some results for fixed point theorems. Harandi [12] extended the iri results in the multi-valued version. But he could only prove the fixed point theorem for multi-valued quasi contraction where contractivity constant $r \in\left[0, \frac{1}{2}\right)$. Haghi [13] proved it for $r \in\left[\frac{1}{2}, 1\right)$. In 2013, Popescu [11] introduced $(s, r)$-contractive multi-valued operator and established its related fixed point theorems. And then, Lingjuan Ye [14] proved the results of weakly $(s, r)$-contractive multi-valued operator for fixed point theorems on b-metric space. Ei Ei Nyein [4] introduced the quasi $(s, r)$-contractive multi-valued operator with contractivity constant $r \in[0,1)$ on metric space and established some results for the related fixed point theorems

2020 Mathematics Subject Classification: 30L99

Received: 18-06-2020, accepted: 03-09-2020. 
on complete metric space. This paper introduces the new type of quasi $(s, r)$ contractive multi-valued operator and extends the results of Ei Ei Nyein [4] on b-complete metric spaces. Some elementary definitions and results will be used throughout this paper in $([1],[3],[6],[7],[14],[15])$.

The following definitions and results from the contractive b-complete metric space are useful to our study. Let $\mathbb{R}^{+}$denote the set of non-negative numbers and $\mathbb{N}$ denote the set of positive integers and $C B(X)$ denote the collection of closed and bounded set of the b-metric space $X$ throughout this paper.

Definition 1.1. [5] Let $X$ be a nonempty set and $K \geq 1$ be a given constant. A function $d: X \times X \rightarrow \mathbb{R}^{+}$is called a b-metric if the following conditions are satisfied:

(1) $d(x, y)=0$ if and only if $x=y$;

(2) $d(x, y)=d(y, x)$, for all $x, y \in X$

(3) $d(x, y) \leq K[d(x, z)+d(z, y)]$, for all $x, y, z \in X$

The pair $(X, d)$ is called a $b$-metric space (with constant $K$ ).

It is noted that a metric space is also a $b$-metric space with $K=1$, but the common is not generally true.

Example 1.2. Let $X=\mathbb{R}^{+}$endowed with a function $d: X \times X \rightarrow \mathbb{R}^{+}$defined by $d(x, y):=|x-y|^{2}$, for all $x, y \in X$. Then $(X, d)$ is a $b$-metric space with constant $K=2$, but it is not a metric space.

Definition 1.3. [16] Let $(X, d)$ be a b-metric space and $\left\{x_{n}\right\}$ be a sequence of $X$ such that

(1) $\left\{x_{n}\right\}$ is covergent if there exists an $x$ in $X$ such that for any $\varepsilon>0$, there exists an $n(\varepsilon) \in \mathbb{N}$, such that $n \geq n(\varepsilon), d\left(x_{n}, x\right)<\varepsilon$.

(2) $\left\{x_{n}\right\}$ is a Cauchy sequence if for any $\varepsilon>0$, there exists an $n(\varepsilon) \in \mathbb{N}$, such that for all $m, n \geq n(\varepsilon), d\left(x_{n}, x_{m}\right)<\varepsilon$.

(3) $(X, d)$ is complete if and only if every Cauchy sequence in $X$ is convergent.

Lemma 1.4. [7] Every sequence $\left\{x_{n}\right\}_{n \in \mathbb{N}}$ of elements from a b-metric space $(X, d)$, having the property that there exists $r \in[0,1)$ such that

$$
d\left(x_{n+1}, x_{n}\right) \leq \operatorname{rd}\left(x_{n}, x_{n-1}\right)
$$

for every $n \in \mathbb{N}$, is Cauchy.

Nalder[10] introduced the Hausdorff metric for multi-valued operator.

Definition 1.5. [14] Let $(X, d)$ be a metric space and $C B(X)$ be the class of all nonempty closed and bounded subsets of $X$. For any $A, B \in C B(X)$, set

$$
H(A, B)=\max \left\{\sup _{x \in A} d(x, B), \sup _{y \in B} d(y, A)\right\},
$$

where $d(x, B)=\inf _{y \in B} d(x, y)$, then $(C B(X), H)$ is a metric space and $H(A, B)$ is called a Hausdorff metric between $A$ and $B$. 
Similarly, if $(X, d)$ is a b-metric space, then $(C B(X), H)$ is a b-metric space. $H(A, B)$ is called a b-Hausdorff metric on $C B(X)$. In the following, unless stated in particular, $H(A, B)$ will denote a b-Hausdorff metric.

Remark 1.6. Suppose that $(X, d)$ is a metric space, then $H(A, B)=0$ iff $A=B$.

Lemma 1.7. [5] Let $(X, d)$ be a b-metric space with the constant $K \geq 1$. For any $A, B, C \in C B(X)$ and any $x, y \in X$ one has the following:

(i) $d(x, B) \leq d(x, b)$, for any $b \in B$;

(ii) $d(x, B) \leq H(A, B)$, for any $x \in A$;

(iii) $H(A, A)=0$;

(iv) $H(A, B)=H(B, A)$;

(v) $H(A, C) \leq K(H(A, B)+H(B, C))$;

(vi) $d(x, A) \leq K(d(x, y)+d(y, A))$

Definition 1.8. [8] Let $(X, d)$ be a b-metric space. An element $x \in X$ is said to be a fixed point of $d: X \rightarrow 2^{X}$ if $x \in T x$, where $2^{X}$ denotes the collection of all non-empty subset of $X$.

Ei Ei Nyein [4] introduced the notion of quasi $(s, r)$-constractive multi-valued operator in metric space as follows:

Definition 1.9. [4] Let $(X, d)$ be a complete metric space and $T: X \rightarrow C B(X)$ be a multi-valued operator. If there exist $r \in[0,1)$ and $s \geq r$ such that

$$
d(y, T x) \leq s d(x, y) \Rightarrow H(T x, T y) \leq r M^{*}(x, y), \forall x, y \in X,
$$

where

$$
M^{*}(x, y)=\max \{d(x, y), d(x, T x), d(y, T y), d(x, T y), d(y, T x)\},
$$

then $T$ is called a quasi $(s, r)$-contractive multi-valued operator on $X$.

Now, we introduce the quasi $(s, r)$-contractive multi-valued operator on the b-complete metric space and extend the results of Ei Ei Nyein [4] in these complete metric space to the results on b-complete metric space.

\section{MAIN RESULTS}

In this section we introduce the notion of quasi $(s, r)$-contractive multi-valued operator on b-metric apace as follows:

Definition 2.1. Let $(X, d)$ be a b-metric space with the constant $K \geq 1$ and $T$ : $X \rightarrow C B(X)$ be a multi-valued operator. If there exists $s, r \in \mathbb{R}$ with $r \in[0,1), s \geq r$ such that for all $x, y \in X$

$$
d(y, T x) \leq K s d(x, y) \Rightarrow H(T x, T y) \leq r M_{T, K}(x, y),
$$

then $T$ is said to be a quasi $(s, r)$-contrative multi-valued operator on $X$ where

$$
M_{T, K}(x, y)=\max \left\{d(x, y), d(x, T x), \frac{1}{2 K} d(y, T y), \frac{1}{2 K} d(x, T y), d(y, T x)\right\} .
$$


The following theorem is a generalization of quasi $(s, r)$-constractive multivalued operator on metric space.

Theorem 2.2. Suppose that $(X, d)$ is a complete b-metric space and $T: X \rightarrow$ $C B(X)$ is a quasi $(s, r)$-contractive multi-valued operator with $r \in[0,1)$ such that $r<\min \left\{\frac{1}{K}, s\right\}$. Then $T$ has a fixed point.

Proof. Let $u_{0} \in X$ and $u_{1} \in T u_{0}$. If $u_{1}=u_{0}$, then $u_{0} \in T u_{0}$. It implies that $u_{0}$ is a fixed point of $T$. If $u_{0} \neq u_{1}$, then we take $u_{2} \in T u_{1}$ such that

$$
d\left(u_{1}, u_{2}\right) \leq H\left(T u_{0}, T u_{1}\right)
$$

Since $d\left(u_{1}, T u_{0}\right)=0 \leq s d\left(u_{1}, u_{0}\right)$. By hypothesis,

$$
\begin{aligned}
H\left(T u_{0}, T u_{1}\right) & \leq r \max \left\{d\left(u_{0}, u_{1}\right), d\left(u_{0}, T u_{0}\right), \frac{1}{2 K} d\left(u_{1}, T u_{1}\right), \frac{1}{2 K} d\left(u_{0}, T u_{1}\right), d\left(u_{1}, T u_{0}\right)\right\} \\
d\left(u_{1}, u_{2}\right) & \leq H\left(T u_{0}, T u_{1}\right) \\
& \leq r \max \left\{d\left(u_{0}, u_{1}\right), d\left(u_{0}, T u_{0}\right), \frac{1}{2 K} d\left(u_{1}, T u_{1}\right), \frac{1}{2 K} d\left(u_{0}, T u_{1}\right), d\left(u_{1}, T u_{0}\right)\right\}, \\
& \leq r \max \left\{d\left(u_{0}, u_{1}\right), d\left(u_{1}, T u_{0}\right), \frac{d\left(u_{0}, u_{1}\right)+d\left(u_{1}, T u_{1}\right)}{2 K}\right\}, \\
& \leq r \max \left\{d\left(u_{0}, u_{1}\right), d\left(u_{1}, T u_{1}\right)\right\} .
\end{aligned}
$$

Case(i) : If $\max \left\{d\left(u_{0}, u_{1}\right), d\left(u_{1}, T u_{1}\right)\right\}=d\left(u_{1}, T u_{1}\right)$, then $d\left(u_{1}, T u_{1}\right) \leq r d\left(u_{1}, T u_{1}\right)$ and it implies that $u_{1} \in T u_{1}$. So $u_{1}$ is a fixed point of $T$.

Case(ii) : If $\max \left\{d\left(u_{0}, u_{1}\right), d\left(u_{1}, T u_{1}\right)\right\}=d\left(u_{0}, u_{1}\right)$, then $d\left(u_{1}, T u_{1}\right) \leq r d\left(u_{0}, u_{1}\right)$. Then there exists $u_{2} \in T u_{1}$ such that $d\left(u_{1}, u_{2}\right) \leq r d\left(u_{0}, u_{1}\right)$. So, one can construct a sequence $\left\{u_{n}\right\} \in X$ such that $u_{n+1} \in T u_{n}$ and

$$
\begin{aligned}
d\left(u_{n+1}, u_{n+2}\right) & \leq r_{1} d\left(u_{n}, u_{n+1}\right), \text { for all } n=0,1,2, \cdots \\
\sum_{n=1}^{\infty} d\left(u_{n}, u_{n+1}\right) & \leq d\left(u_{0}, u_{1}\right)+r_{1} d\left(u_{0}, u_{1}\right)+r_{1}^{2} d\left(u_{0}, u_{1}\right)+\cdots \\
& \leq \frac{1}{1-r_{1}} d\left(u_{0}, u_{1}\right) \\
& <\infty .
\end{aligned}
$$

Therefore, $\left\{u_{n}\right\}$ is a Cauchy sequence and for the completeness of $X$, there is a point $u^{*} \in X$ such that $\lim _{n \rightarrow \infty} u_{n}=u^{*}$.

Now we show that there exists a subsequence $\left\{u_{n_{p}}\right\}$ of $\left\{u_{n}\right\}$ such that $d\left(u^{*}, T u_{n_{p}}\right) \leq K s d\left(u^{*}, u_{n_{p}}\right)$.

Assume that there exists a positive integer $n_{1} \in \mathbb{N}$ such that

$$
d\left(u^{*}, T u_{n_{p}}\right)>K s d\left(u^{*}, u_{n}\right), \quad \forall n \geq n_{1} .
$$

By induction,

$$
d\left(u^{*}, u_{n+p}\right)>(K s)^{p} d\left(u^{*}, u_{n}\right), \quad \forall n \geq n_{1}, p \geq 1,
$$


Moreover,

$$
\begin{aligned}
d\left(u_{n}, u_{n+p}\right) & \leq K\left[d\left(u_{n}, u_{n+1}\right)+d\left(u_{n+1}, u_{n+p}\right)\right] \\
& \leq K d\left(u_{n}, u_{n+1}\right)+K\left[r_{1} d\left(u_{n}, u_{n+1}\right)+r_{1} d\left(u_{n+1}, u_{n+p-1}\right)\right] \\
& \leq\left(\frac{1-\left(K r_{1}\right)^{p}}{1-K r_{1}}\right) d\left(u_{n}, u_{n+1}\right), \quad \forall n \geq n_{1}, p \geq 1 .
\end{aligned}
$$

Set $p \rightarrow \infty$,

$$
d\left(u^{*}, u_{n}\right) \leq \frac{1}{1-K r_{1}} d\left(u_{n}, u_{n+1}\right), n=0,1,2, \cdots
$$

So

$$
d\left(u^{*}, u_{n}\right) \leq \frac{\left(K r_{1}\right)^{p}}{1-K r_{1}} d\left(u_{n}, u_{n+1}\right), \quad \forall n \geq n_{1}, p \geq 1 .
$$

By the equation (1) and (2), we have

$$
(K s)^{p} d\left(u^{*}, u_{n}\right) \leq\left(\frac{\left(\frac{r_{1}}{s}\right)^{p}}{1-K r_{1}}\right) d\left(u_{n}, u_{n+1}\right) .
$$

Set $p \rightarrow \infty$, we obtain

$$
d\left(u^{*}, u_{n}\right)=0, \forall n>n_{1},
$$

which is contradict to (1). Therefore there exists a subsequence $\left\{u_{n_{p}}\right\}$ of $\left\{u_{n}\right\}$ such that

$$
d\left(u^{*}, T u_{n_{p}}\right) \leq K s d\left(u^{*}, u_{n_{p}}\right)
$$

Thus,

$d\left(u_{n_{p+1}}, T u^{*}\right) \leq H\left(T u^{*}, T u_{n_{p}}\right)$

$\leq r \max \left\{d\left(u^{*}, u_{n_{p}}\right), d\left(u^{*}, T u^{*}\right), \frac{1}{2 K} d\left(u_{n_{p}}, T u_{n_{p}}\right), \frac{1}{2 K} d\left(u^{*}, T u_{n_{p}}\right), d\left(u_{n_{p}}, T u^{*}\right)\right\}$.

Set $p \rightarrow \infty$, we have

$$
d\left(u^{*}, T u^{*}\right) \leq r d\left(u^{*}, T u^{*}\right)
$$

It implies $u^{*} \in T u^{*}$ and the proof is completed.

Corollary 2.3. Let $(X, d)$ be a complete $b$-metric space and $T: X \rightarrow X$ be a $(s, r)$-contractive single-valued operator with $r<\min \left\{\frac{1}{K}, s\right\}$. Then $T$ has a fixed point. Moreover, if $K r<1 \leq K s$ and $r \in[0,1)$, the fixed point is unique.

Proof. If $T: X \rightarrow X$ is a single-valued contractive operator, using Theorem 2.3, there exists a fixed point. Assume that there are two different fixed points $u^{*}$ and $w^{*}$ such that $T w^{*}=w^{*}$ and $T u^{*}=u^{*}$.

Then

$$
d\left(u^{*}, T w^{*}\right)=d\left(u^{*}, w^{*}\right) \leq K s d\left(w^{*}, T u^{*}\right) .
$$

So, $d\left(T u^{*}, T w^{*}\right)$ 
$\leq r \max \left\{d\left(u^{*}, w^{*}\right), d\left(u^{*}, T u^{*}\right), \frac{1}{2 K} d\left(w^{*}, T w^{*}\right), \frac{1}{2 K} d\left(u^{*}, T w^{*}\right), d\left(w^{*}, T u^{*}\right)\right\}$.

Thus

$$
\begin{aligned}
d\left(u^{*}, w^{*}\right) & \leq r \max \left\{d\left(u^{*}, w^{*}\right), d\left(u^{*}, T u^{*}\right), \frac{1}{2 K} d\left(w^{*}, T w^{*}\right), \frac{1}{2 K} d\left(u^{*}, T w^{*}\right), d\left(w^{*}, T u^{*}\right)\right\} \\
& =r \max \left\{d\left(u^{*}, w^{*}\right), \frac{1}{2 K} d\left(u^{*}, T w^{*}\right)\right\} \\
& \leq r d\left(u^{*}, w^{*}\right) .
\end{aligned}
$$

It is contradict to $u^{*} \neq w^{*}$ and this completes the proof.

Theorem 2.4. Let $(X, d)$ be a complete b-metric space and $T: X \rightarrow C B(X)$ be a quasi $(s, r)$ - contractive multi-valued operator. If there exist $s, r \in[0,1)$ such that $r<s<\frac{1}{K}$ with

$\frac{1}{1-K r} d(x, T x) \leq d(x, y) \leq \frac{1}{1+K s} d(x, T x) \Rightarrow H(T x, T y) \leq r M_{T, K}(x, y), \quad \forall x, y \in X$

where $M_{T, K}(x, y)=\max \left\{d(x, y), d(x, T x), \frac{1}{2 K} d(y, T y), \frac{1}{2 K} d(x, T y), d(y, T x)\right\}$.

Then $T$ has a fixed point.

Proof. Let $u_{0} \in X$ and $u_{1} \in T u_{0}$ such that $d\left(u_{0}, u_{1}\right) \leq \frac{1-K r_{1}}{1-K s} d\left(u_{0}, T u_{0}\right)$, where $0 \leq r<r_{1}<s$. Then we have

$$
\begin{aligned}
\frac{1}{1+K r} d\left(u_{0}, T u_{0}\right) & \leq d\left(u_{0}, T u_{0}\right) \\
& \leq d\left(u_{0}, u_{1}\right) \\
& \leq \frac{1}{1-K s} d\left(u_{0}, T u_{0}\right)
\end{aligned}
$$

By our hypothesis, we have

$$
\begin{aligned}
d\left(u_{1}, u_{2}\right) & \leq d\left(u_{0}, T u_{1}\right) \\
& \leq H\left(T u_{0}, T u_{1}\right) \\
& \leq r \max \left\{d\left(u_{0}, u_{1}\right), d\left(u_{0}, T u_{0}\right), \frac{1}{2 K} d\left(u_{0}, T u_{1}\right), \frac{1}{2 K} d\left(u_{1}, T u_{1}\right), d\left(u_{1}, T u_{0}\right)\right\} \\
& \leq r \max \left\{d\left(u_{0}, u_{1}\right), d\left(u_{0}, T u_{0}\right), \frac{d\left(u_{0}, u_{1}\right)+d\left(u_{1}, u_{2}\right)}{2}\right\} \\
& \leq r \max \left\{d\left(u_{0}, u_{1}\right), d\left(u_{1}, u_{2}\right)\right\} .
\end{aligned}
$$

Case(i) : If $\max \left\{d\left(u_{0}, u_{1}\right), d\left(u_{1}, u_{2}\right)\right\}=d\left(u_{1}, u_{2}\right)$, then $d\left(u_{1}, u_{2}\right)=0$. It implies $u_{1}$ is a fixed point of $T$.

Case(ii) : If $\max \left\{d\left(u_{0}, u_{1}\right), d\left(u_{1}, u_{2}\right)\right\}=d\left(u_{0}, u_{1}\right)$, then

$$
d\left(u_{1}, u_{2}\right) \leq r d\left(u_{0}, u_{1}\right), r<1 .
$$

Then there exists $u_{2} \in T u_{1}$ such that $d\left(u_{1}, u_{2}\right) \leq r_{1} d\left(u_{0}, u_{1}\right)$ and $d\left(u_{1}, u_{2}\right) \leq$ $\frac{1-K r_{1}}{1-K s} d\left(u_{1}, T u_{1}\right)$. Therefore, one can construct the sequence $\left\{u_{n}\right\}$ in $X$ such that $u_{n} \in T u_{n-1}$ with 
$d\left(u_{n}, u_{n+1}\right) \leq r_{1} d\left(u_{n-1}, u_{n}\right)$ and $d\left(u_{n-1}, u_{n}\right) \leq \frac{1-K r_{1}}{1-K s} d\left(u_{n-1}, T u_{n-1}\right)$, for $n=$ $1,2, \cdots$.

$$
\begin{aligned}
\sum_{n=0}^{\infty} d\left(u_{n}, u_{n+1}\right) & =d\left(u_{0}, u_{1}\right)+d\left(u_{1}, u_{2}\right)+d\left(u_{2}, u_{3}\right)+\cdots \\
& \leq\left[1+r_{1}+r_{1}^{2}+r_{1}^{3}+\cdots\right] d\left(u_{0}, u_{1}\right) \\
& \leq \frac{1}{1-r_{1}} d\left(u_{0}, u_{1}\right) \\
& <\infty
\end{aligned}
$$

So, $\left\{u_{n}\right\}$ is a Cauchy sequence and there is a point $u^{*} \in X$ such that $\lim _{n \rightarrow \infty} u_{n}=$ $u^{*}$ since $X$ is b-complete.

Again,

$$
\begin{aligned}
d\left(u_{n-1}, u_{n-1+p}\right) & \leq K\left[d\left(u_{n-1}, u_{n}\right)+d\left(u_{n}, u_{n-1+p}\right)\right] \\
& \leq K d\left(u_{n-1}, u_{n}\right)+K\left[K d\left(u_{n}, u_{n+1}\right)+K d\left(u_{n+1}, u_{n-1+p}\right)\right] \\
& \leq\left[K r_{1}+\left(K r_{1}\right)^{2}+\left(K r_{1}\right)^{3}+\cdots+\left(K r_{1}\right)^{p-1}\right] d\left(u_{n-1}, u_{n}\right) \\
& \leq\left(\frac{1-\left(K r_{1}\right)^{p}}{1-K r_{1}}\right) d\left(u_{n-1}, u_{n}\right), \quad \forall p \geq 1, n \geq n_{1} \in \mathbb{N} .
\end{aligned}
$$

Letting $p \rightarrow \infty$, we get

$$
d\left(u^{*}, u_{n-1}\right) \leq \frac{1}{1-K r_{1}} d\left(u_{n-1}, u_{n}\right), \quad \forall n \geq 0 .
$$

Since $d\left(u_{n-1}, u_{n}\right) \leq \frac{1-K r_{1}}{1-K s} d\left(u_{n-1}, T u_{n-1}\right)$, it follows that

$$
d\left(u^{*}, u_{n-1}\right) \leq \frac{1}{1-K s} d\left(u_{n-1}, T u_{n-1}\right), \quad \forall n \geq 0 .
$$

Suppose that there is a positive integer $n_{1}$ such that

$$
d\left(u^{*}, u_{n-1}\right)<\frac{1}{1+K r} d\left(u_{n-1}, T u_{n-1}\right), \quad \forall n \geq n_{1} \in \mathbb{N} .
$$

Then we obtain

$$
\begin{aligned}
d\left(u_{n-1}, u_{n}\right) & \leq K\left[d\left(u_{n-1}, u^{*}\right)+d\left(u^{*}, u_{n}\right)\right] \\
& <\frac{K}{1+K r}\left[d\left(u_{n-1}, T u_{n-1}\right)+d\left(u_{n}, T u_{n}\right)\right] \\
& <\frac{K}{1+K r}\left[d\left(u_{n-1}, T u_{n-1}\right)+r K d\left(u_{n-1}, T u_{n}\right)\right] .
\end{aligned}
$$

It implies $d\left(u_{n-1}, u_{n}\right)<K d\left(u_{n-1}, T u_{n-1}\right)$ which is impossible. Thus, there is a subsequence $\left\{u_{n-1_{p}}\right\}$ of $\left\{u_{n-1}\right\}$ such that

$$
d\left(u^{*}, u_{n-1_{p}}\right) \geq \frac{1}{1+K r} d\left(u_{n-1}, T u_{n-1_{p}}\right), \quad \forall p \geq n_{1} \in \mathbb{N} .
$$

Since

$$
d\left(u^{*}, u_{n-1}\right) \leq \frac{1}{1-K s} d\left(u_{n-1}, T u_{n-1}\right), \quad \forall n \geq 0 .
$$


It follows that $\left.H\left(T u^{*}, T u_{n-1_{p}}\right)\right) \leq r M_{T, K}\left(\left(u^{*}, u_{n-1_{p}}\right)\right.$.

It implies

$d\left(u^{*}, u_{n-1_{p}}\right)$

$\leq r \max \left\{d\left(u^{*}, u_{n-1_{p}}\right), d\left(u^{*}, T u^{*}\right), \frac{1}{2 K} d\left(u_{n-1_{p}}, T u_{n-1_{p}}\right), \frac{1}{2 K} d\left(u^{*}, T u_{n-1_{p}}\right), d\left(u_{n-1_{p}}, T u^{*}\right)\right\}$

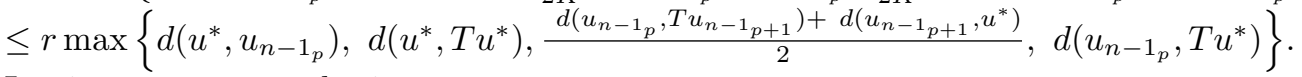

Letting $p \rightarrow \infty$, we obtain

$$
d\left(u^{*}, T u^{*}\right) \leq r \max \left\{d\left(u^{*}, T u^{*}\right), \frac{d\left(u^{*}, T u^{*}\right)}{2}\right\} .
$$

So, $d\left(u^{*}, T u^{*}\right)=0$ and hence $u^{*} \in T u^{*}$.

Corollary 2.5. Let $(X, d)$ be a complete b-metric space and $T: X \rightarrow X$ be a single value operator. Assume that there exists $r \in[0,1)$ such that $K r<1$ with

$$
\frac{1}{1+K r} d(x, T x) \leq d(x, y) \leq \frac{1}{1-K r} d(x, T x) \Rightarrow H(T x, T y) \leq r M_{T, K}(x, y),
$$

where $M_{T, K}(x, y)=\max \left\{d(x, y), d(x, T x), \frac{1}{2 K} d(y, T y), \frac{1}{2 K} d(x, T y), d(y, T x)\right\}$. Then $T$ has a fixed point.

Proof. One can easily prove that for $u_{0} \in X$, the sequence $\left\{u_{n}\right\}$ defined by $u_{n+1} \in$ $T u_{n}$ satisfies the relationship $d\left(u_{n+1}, u_{n+2}\right) \leq r d\left(u_{n}, n_{n+1}\right)$ for $n=0,1,2, \cdots$. So the sequence $\left\{u_{n}\right\}$ is Cauchy and since $X$ is b-complete. There is a $u^{*}$ in $X$ such that $\lim _{n \rightarrow \infty} u_{n}=u^{*}$. As in the proof of theorem 2.4, we can prove that

$$
d\left(u^{*}, u_{n}\right) \leq \frac{1}{1-K r} d\left(u_{n}, u_{n+1}\right), \text { for } n=0,1,2, \cdots
$$

and there exists a subsequence $\left\{u_{n_{p}}\right\}$ of $\left\{u_{n}\right\}$ such that $d\left(u^{*}, u_{n_{p}}\right) \geq \frac{1}{1+K r} d\left(u_{n_{p}}, u_{n_{p+1}}\right)$ holds for every $p \geq n_{1} \in \mathbb{N}$.

So, we have

$d\left(u_{n_{p+1}}, T u^{*}\right)$

$\leq r \max \left\{d\left(u^{*}, u_{n_{p}}\right), d\left(u^{*}, T u^{*}\right), \frac{1}{2}\left[d\left(u^{*}, T u_{n_{p+1}}\right)+d\left(u_{n_{p}}, T u^{*}\right)\right], d\left(u_{n_{p}}, T u^{*}\right)\right\}$

$\leq r \max \left\{d\left(u^{*}, u_{n_{p}}\right), d\left(u^{*}, T u^{*}\right), d\left(u^{*}, u_{n_{p+1}}\right), d\left(u_{n_{p}}, T u^{*}\right)\right\}$.

As $p \longrightarrow \infty$, we obtain

$$
d\left(u^{*}, T u^{*}\right) \leq r d\left(u^{*}, T u^{*}\right)
$$

and it implies $u^{*}=T u^{*}$.

\section{APPLICATION}

Fixed point theorems are useful to apply in differential equations and integral equations.

Let $X=\{f \mid f:[a, b] \rightarrow \mathbb{R}$ is continuous $\}$. Then $(X, d)$ be a b-complete metric space if we define

$$
d(x, y)=\max _{t \in[a, b]}|x(t)-y(t)|^{2} \quad \forall x, y \in X .
$$


Consider the differential equation

$$
\left\{\begin{array}{l}
\left.\frac{d y}{d x}=f(x, y)\right) \\
y\left(x_{0}\right)=y_{0}
\end{array}\right.
$$

It is equivalent to the integral equations

$$
y(x)=y_{0}+\int_{x_{0}}^{x} f(x, y(t)) d t .
$$

We choose a constant $\delta$ such that $0<\delta<1$, and define the operator $T: C[a, b] \rightarrow \mathbb{R}$ by

$$
T y(x)=y_{0}+\int_{x_{0}}^{x} f(x, y(t)) d t .
$$

where $C[a, b] \subset X$.

Then the integral equations have a solution is equivalent to the operator $T$ has a fixed point. Assume that there exist $r \in[0,1), s>0$ and $r<\left\{\frac{1}{2}, s\right\}$, such that for all $y_{1}, y_{2} \in X$, we have

$$
\left|y_{2}-\left[y_{0}+\int_{x_{0}}^{x} f(x, y(t)) d t\right]\right|^{2} \leq 2 s\left|y_{1}-y_{2}\right|^{2}
$$

It implies that $\left|f\left(z, y_{1}\right)-f\left(z, y_{2}\right)\right|^{2} \leq r\left|y_{1}-y_{2}\right|^{2}$.

We have

$$
\begin{aligned}
d\left(T y_{1}, T y_{2}\right) & =\max _{\left|x-x_{0}\right|<\delta} \mid \int_{x_{0}}^{x}\left[f\left(t, y_{1}(t)\right)-\left.f\left(t, y_{2}(t)\right] d t\right|^{2}\right. \\
& \leq \max _{\left|x-x_{0}\right|<\delta} \int_{x_{0}}^{x} r\left|y_{1}(t)-y_{2}(t)\right|^{2} d t \\
& \leq r \delta \max _{\left|t-x_{0}\right|<\delta}\left|y_{1}(t)-y_{2}(t)\right|^{2} \\
& =r \delta d\left(y_{1}(t), y_{2}(t)\right) \\
& \leq r \max \left\{d\left(y_{1}, y_{2}\right), d\left(y_{1}, T y_{1}\right), \frac{1}{4} d\left(y_{2}, T y_{2}\right), \frac{1}{4} d\left(y_{1}, T y_{2}\right), d\left(y_{2}, T y_{1}\right)\right\}
\end{aligned}
$$

Then the operator $T$ satisfies the conditions of Theorem 2.3. Hence $T$ has a fixed point and there exists a continuous function $y_{0}(t)$ such that

$$
y_{0}(t)=y_{0}+\int_{x_{0}}^{x} f\left(x, y_{0}(t)\right) d t, \forall x \in\left[x_{0}-\delta, x_{0}+\delta\right] .
$$

\section{REFERENCES}

[1] S.L. Singh, S. Czerwik, K. Kr ol, A. Singh,, Coincidences and fixed points of hybrid contractions, Tamsui Oxf. J. Math. Sci., 24, (2008), 401416.

[2] S. Czerwik, Contraction mappings in b-metric spaces, Acta Math. Inform. Univ. Ostraviensis, $1,(1993), 5-11$.

[3] S. Czerwick, Nonlinear set-valued contraction mappings in b-metric spaces, Atti del Seminario Matematico e Fisico dellUniversit'a di Modena, 46, (1998), 263276.

[4] Ei Ei Nyein, Dianlu Tian, Aung Khaing Zaw, Quasi (s,r)-Contractive Multi-Valued Operators and Related Fixed Point Theorems, Mathematics, 2020, 8(1), 64; doi:10.3390/math8010064. 
[5] M. Jleli, B. Samet, C. Vetro, F. Vetro, Fixed points for multivalued mappings in b-metric spaces, Abstract and Applied Analysis, 2015 (2015), 1-7.

[6] William Kirk, Naseer Shahzad, Fixed Point Theory in Distance Spaces, Springer, New York, (2014).

[7] Radu Miculescu, Alexandru Mihail, New fixed point theorems for set-valued contractions in b-metric spaces, arXiv:1512.03967v1 [math.CA] 12, Dec (2015).

[8] H. Aydi, M.F. Bota, E. Karapinar, S. Mitrovic, A fixed point theorem for set-valued quasicontractions in b-metric spaces, Fixed Point Theory and Applications, 2012, (2012): 88

[9] L.B. Ciric, A generalization of Banachs contraction principle, Proc. Amer. Math. Soc., 45 (2) (1974), 267273

[10] S.B. Nadler, Multi-valued contraction mappings, Pacific J. Math., 30 (1969), 475488.

[11] O. Popescu, A new type of contractive multivalued operators, Bull. Sci. Math., 137, (2013), $30-44$.

[12] A. Amini-Harandi, Fixed point theory for set-valued quasi-contraction maps in metric spaces, Appl. Math. Lett., 24, (2011), 1791-1794.

[13] R.H. Haghi,S. Rezapour, N. Shahzad, On fixed points of quasi-contraction type multifunctions, Appl. Math. Lett., 25, (2012), 843-846.

[14] Lingjuan Ye, Congcong Shen, weakly (s,r)-contractive multi-valued operators on b-mettric space, J.Nonlinear Sci. Appl., 11 (2018), 358-367.

[15] J.R. Roshan, V. Parvaneh, Z. Kadelburg, Common fixed point theorems for weakly isotone increasing mappings in ordered b-metric spaces, J. Nonlinear Sci. Appl., 7 (2014), 229245.

[16] M. Boriceanu, M. Bota, A. Petrusel, Multivalued fractals in b-metric spaces, Cent. Eur. J. Math., 8 (2010), 367-377. 